\title{
EVALUATION OF FUNDUS FIRST LAPAROSCOPIC CHOLECYSTECTOMY IN CASES OF ACUTE CHOLECYSTITIS: THEODOR BILHARZ RESEARCH INSTITUTE EXPERIENCE
}

\author{
By \\ MOHAMED ABBAS*, AHMED MOHAMED ABDELAAZIZ HASSAN, \\ YOUSSEF F. YOUSSEF and HESHAM ABD ALAZIZ
}

Department of General Surgery, Theodor Bilharz Research Institute, P.O Box 30, Imbaba 12411, Giza, Egypt ("correspondence: <mabbas_mahmoud@yahoo.com)

\begin{abstract}
This study included 125 patients with acute cholecystitis of them 29 were males. All were subjected to fundus-first (FF) laparoscopic cholecystectomy. The mean operative time was $63 \pm 8$ minutes (range 37-95 minutes). There were 4 cases of prolonged post-operative bile leakage, 3 of them stopped spontaneously within one week, and last one needed an endoscopic biliary stent. Gall bladder perforation with peritoneal contamination occurred in 6 patients. Laparoscopic trans-choledochal CBD exploration was indicated in 3 patients due to residual stone in intra-operative cholangiography. Conversion to open cholecystectomy was acquired in 4 patients the mean hospital stay was 1.6 days (range 6 hours to 5 days) except those with post-operative bile leakage that stayed from 8-14 days. Key words: acute
\end{abstract} cholecystitis, laparoscopic cholecystectomy,

\section{Introduction}

The acute cholecystitis results from obstruction of the cystic duct, usually by a gallstone, followed by distension and subsequent chemical or bacterial inflammation of the gallbladder. People with acute cholecystitis usually have unremitting right upper quadrant pain, anorexia, nausea, vomiting, and fever. About $95 \%$ of people with acute cholecystitis have gallstones (calculous cholecystitis) \& 5\% lack gallstones (acalculous cholecystitis). Severe acute cholecystitis can lead to necrosis of the gallbladder wall or gangrenous cholecystitis (Halpin, 2014). The introduction of laparoscopic cholecystectomy in surgical practice was pioneered by Mouret (1991). Laparoscopic cholecystectomy (LC) is one of the commonest surgical procedures in worldwide (Bayrak and Altıntas, 2018). It becomes the most standard procedure for management of the symptomatic cholelithiasis or acute cholecystitis in patients without and specific contra-indications. The most significant LC morbidity is bile duct injury, which implies not only complex procedures of repair, but also a serious impact on patients' outcomes (Zha et al, 2010; Neri et al, 2013). The fundus-first (FF) technique (dome down, antegrade dissection) decreases the rate of major compl- cations and conversion rate in cases with difficult dissection of the cystic duct and cystic artery (Elshaer et al, 2015). Although, this technique is feasible, yet not widely practiced with its true role would seem, as yet, to be undefined.

This study aimed to assess the feasibility, safety, and outcome of the fundus first laparoscopic cholecystectomy in cases of acute cholecystitis

\section{Patients and methods}

From January 2013 to March 2017, 125 laparoscopic cholecystectomies were performed at Theodor Bilharz Research Institute, for acute cholecystitis. In the present laparoscopic experience, a change of surgical technique was introduced, so that fundus first dissection replaced retrograde dissection. Patients recruited for the study was those with acute cholecystitis defined as follows: 1- Clinically: had upper right quadrant pain and tenderness with rebound pain in some cases, chills before fever. 2- Laboratorial: had leukocytosis; most of them suffered from increase in SGOT/SGPT (Metwaly et al, 2012), and 3-Radiologically: abdominal US evaluation showed signs of local phlogosis of the gallbladder characterized by an increase in the thickness of the gallbladder wall (more than $6 \mathrm{~mm}$ ) associated 
in some cases with empyema and pericholecystic fluid collection.

All patients underwent laparoscopic cholecystectomy within 96 hours post demission.

Ethical approval: The study was complied with all the relevant national regulations, institutional policies and in accordance the tenets of the Helsinki Declaration, and has been approved by the authors' institutional review board or equivalent committee. The informed consent has been obtained from all individuals included in this study.

Statistical analysis: The operative time, conversion to open surgery, and the gallbladder perforation with peritoneal contamination, hemorrhages, bile duct (BD) injury, residual CBD stones attributable to the mobilization of little stones and iatrogenic bowel perforation were recorded and analyzed.

Surgical technique: The patient was lying supine and the surgeon was positioned on the patient's left side (North American Positioning). The camera operator stands on the patient's left and to the left of the surgeon, while the assistant stands on the patient's right. The video monitor was positioned on the patient's right above the level of the costal margin. Exposure can be improved by tilting the patient in the reverse Trendelenburg position and rotating the table with the patient's right side up. Gravity pulls the duodenum, the colon, and the omentum away from the gallbladder, thereby increasing the working space available in the upper abdomen. Either an open or closed technique was used to establish the pneumoperitoneum. A 10-mm trocar was inserted through the supraumbilical incision and a 30 degree telescope was then inserted through the umbilical port, and an examination of the peritoneal cavity was performed. A $10-\mathrm{mm}$ operating port was placed subxiphoid, and two additional 5-mm trocars were positioned subcostally in the right upper quadrant in the midclavicular and anterior axillary lines. A diathermy hook was used to separate the fundus from the liver, leaving a peritoneal rim on the liver edge. The peritoneal rim, which was usually thick in such cases, was then used to retract the liver cranially. The assisted retraction of liver was performed using the liver retractor if needed. The gall bladder was separated from the liver using blunt dissection. A densely adherent gallbladder may occasionally necessitate the use of a diathermy hook. Particular attention was given to hemostasis in each step. If a large Hartmann's pouch stone was making the dissection difficult, it was either dislodged to the body of gall bladder, or the pouch was opened away from the neck of the gall bladder and the stone was removed. This facilitated the lateral retraction of Hartmann's pouch to complete its separation from the cystic duct and/or the bile duct and expose the area of the cystic duct pedicle.

The cystic duct and cystic artery were then separated close to the neck of the gall bladder which was double clipped. Intra-operative cholangiography was done either transcholecystic or trans-cystic duct according to the case.

\section{Results}

The 125 patients were diagnosed as acute cholecystitis, operated upon with fundus first laparoscopic cholecystectomy during the period from January 2013 to March 2017. 96 patients were females while 29 were males with an average age of 24-81 years old. 73 patients were diabetic in which 26 was insulin dependent and 47 were on oral hypoglycemic. Liver cirrhosis was confronted in 27 patients as it is epidemic in Egypt, 21 patients were Child A and 6 patients were Child B according to ChildPugh score for liver diseases. 19 patients had previous ERCP for CBD choledechlithiasis with an average time interval from ERCP of 2-7 days. Laparoscopic trans-choledochal CBD exploration was needed in 3 patients due to residual stone appeared in intraoperative cholangiography. Conversion to open cholecystectomy was acquired in 4 patients, 2 of them had very fibrous and contracted GB with the co-existing liver cirrhosis, one patient with Mirizzi type III in 
which conversion was essential to deal with CBD fistula; while the last patient, who was also cirrhotic, conversion was to deal with bleeding from an aberrant artery. Bleeding from the gallbladder bed in the liver was controlled by pressure and absorbable hemostatic materials. The cystic artery proper was often not clearly seen due to fibrosis and diathermy dissection close on the gallbladder wall. On several occasions moderate bleeding from the artery occurred near the neck of the gallbladder that was relatively straightforward to control using clips or diathermy. Gall bladder perforation with peritoneal contamination occurred in 6 patients, 4 were induced by surgeon to extract an impacted stone in the Hartmann pouch to facilitate the dissection, however the contamination did not affect the procedure and managed by copious saline irrigation. Management of the cystic duct was individualized according to the surgeon and included use of titanium clips (111 patients), intracorporeal suturing (9 patients) and endoloops (2 patients). Residual choledochlithiasis happened in 2 patients whom required post-operative ERCP. Drains were routinely used except in 15 cases in which the operative field was completely dry. Four cases were of prolonged post-operative bile leakage, 3 of them stopped spontaneously within a week, and remaining one was more prolonged and was subjected to ERCP that revealed lateral common hepatic duct tear to which an endoscopic biliary stent was curative. There was no reported bowel perforation during the procedure. The mean operative time was $63 \pm 8$ minutes (range $37-95$ minutes) and the mean hospital stay was 1.6 days (range 6 hours to 5 days) except those with postoperative bile leakage that stayed from 8- 14 days with an average of $10 \pm 3.9$ day.

\section{Discussion}

Laparoscopic cholecystectomy (LC) was first introduced by Eric Muhe through a direct-view laparoscope without any camera monitor imaging system in 1985 (Reynolds 2001). In the mid-1990s, Kato et al. (1996) reported that the gallbladder could be successfully separated from the cystic bed via dissection of the Calot's triangle. Since then, the retrograde approach has become widely used by surgeons throughout the world, and LC became the standard treatment for the gallstone disease and the acute cholecystitis. In the setting of difficult dissection of Calot's triangle during LC, the risk of severe complications and the rate of conversion to open surgery increases. Although conversion to open surgery is not considered as a failure, it is clear that it eliminates the advantages of laparoscopy and lengthens the time of recovery and does not always provide a better view of the anatomy (Henneman et al, 2013).In the era of minimally invasive surgeries, junior surgeons, in particular, do not have enough experience with the open approach. This may lead to more serious bile duct injuries, such as transection or resection of CBD (Wolf et al, 2009). The standard technique was the grasper on the fundus of the gallbladder to apply cephalad traction to elevate the liver to expose Calot's triangle for dissection (McIntyre et al, 1996). Hunter brought attention to the importance of lateral traction on the neck of the gallbladder to open out Calot's triangle and Strasberg's writings on the critical view of safety have been influential (Hunter 1991; Strasberg 2002). Intraoperative cholangiography and use of $30^{\circ}$ laparoscope may play some role in avoiding bile duct injuries (BDI) and allowing the safer LC (Hugh 1997; Hugh 2002). There are some cases where standard retraction fails to expose Calot's triangle or allow safe dissection. In such cases, the laparoscopic liver retractors are now readily available and the gallbladder can be mobilized fundus first whilst the liver is kept elevated by a retractor. Despite this even relatively recent influential articles have stated that the fundus first technique is difficult to apply in LC because of loss of traction on the liver when the fundus is mobilized (Hugh 2002). While, the FF tech- 
nique is feasible it was not widely practiced, therefore an analysis of unselected LCs carried out by the experienced laparoscopic surgeon using modern instrumentation that seemed worthwhile. In cases of difficulty in dissection and inability in determining the biliary duct and/or cystic artery, the option of FF should be considered before conversion. The conversion rates to open surgery during LC range between $1 \%$ and $24 \%$ (Lo et al, 1997; Araujo-Teixeira et al, 1999; Mattioli et al, 1999; Parra Blanco et al, 1999). The conversion rate can be as high as $44 \%$ during $\mathrm{LC}$ in patients with acute gangrenous cholecystitis (Koperna et al, 1999). Many authors confirmed feasibility FF in patients with the acute or chronic inflammation recommending that it might decrease the BDI rate (Sekimoto et al, 1998; Raj et al, 2001; Ota et al, 2003; Rosenberg and Leinskold 2004). In addition, several authors have reported that FF helps to avoid open surgery. The use of the fundus-first dissection in difficult cases decreased the conversion rate from a potential $5.2 \%$ to $1.2 \%$ (Mahmud et al, 2002). Similarly Gupta et al. (2004) reported a decrease in conversion rate in a small series of patients with chronic cholecystitis from $18.8 \%$ to 2.1\%. Palanivelu et al. (2006) reported 265 LCs in cirrhotic patients and noted that liver retraction was needed in some cases to allow exposure of Calot's triangle and that FF was resorted to in $8.3 \%$ of cases. Ainslie et al. (2000) noted that liver retraction and FF confers an advantage in difficult cholecystectomies due to the opens angle between the cystic duct and bile duct and contributed to their low conversion rate with no BDI. Tuveri et al. (2008) reported a large series where FF was used in $1.5 \%$ of cases due to difficult anatomy in Calot's triangle with a success rate of $80 \%$. Others recommended routine use of FF rather than reserving it for difficult cases. Cengiz et al. (2005) randomized 80 elective patients to compare the two dissection techniques and found that FF combined with ultrasonic dissection were quicker and associated with less nausea and pain. Ichihara et al. (2004) reported that tape ligature of the cystic duct then fundus first dissection in 500 patients and recommended it as a way of decreasing rates of BDI. Yamakawa et al. (2007) described a case where they felt that FF avoided a BDI in a patient with aberrant biliary anatomy. Wang et al. (2006) presented a series showing that FF was safe and effective in elderly patients with acute cholecystitis. Neri et al. (2013) reported that FF reduced the operative time and was an easier technique to perform. However, the fact that most surgeons do not use FF routinely shows that it is a more complex operation and is in keeping with the principle of Ockham's razor, that the simplest solution is the best. The FF technique might pose some technical difficulties during LC. Retracting the liver can be difficult during cholecystectomy when using this technique. Therefore, we preferred to leave a part of peritoneum on the liver bed for retraction to overcome this problem. The other limitation of the FF technique is the blood staining of the operative field in case of bleeding that might increase the rate of conversion or bile duct injury (Koperna et al, 1999). The flow direction of the bleeding is to the opposite site of dissection direction in retrograde dissection, whereas blood flows directly to the dissection field in the FF technique. Therefore, attentive hemostasis is crucial to avoid blood staining of the dissection field. The present study reported more favorable conversion and bile leakage rates compared to other studies (Strasberg and Dirk, 2012). The use of FF technique might affect the duration of surgery. In the present study, the operative time in severely inflamed gall bladders was longer than in the averagely inflamed cases. These agreed with the other authors who also reported in their comparative study a time and a conversion rate advantages of FF technique compared to conventional one in severely inflamed cases (Gupta, 2004). The gall bladder perforation 
during separation from the liver did not add more morbidity or affect the postoperative hospital stay. There was no bowel perforation during the procedure without mortality.

\section{Conclusion}

No doubt, the early laparoscopic cholecystectomy is usually associated with reduced hospital stay, sick leave, and health care expenditures. Fundus first laparoscopic cholecystectomy is a feasible and safe technique in cases of acute cholecystitis. It can be proposed as a standard technique mainly in severely inflamed cases. However, this must not lower the threshold of conversion to open surgery when indicated.

\section{References}

Ainslie, WG, Larvin, M, Martin, IG, McMahon, MJ, 2000: Liver retraction techniques for laparoscopic cholecystectomy. Surg. Endosc. 14: 311-8.

Araujo-Teixeira, P, Rocha-Reis, J, Costa-Cabral, A, Barros, H, Saraiva, AC, et al, 1999: Laparoscopy or laparotomy in acute cholecystitis (200 cases): Comparison of the results and factors predictive of conversion. Chirurgie 124: 529-35.

Bayrak, M, Altıntas, Y, 2018: Comparing laparoscopic cholecystectomy in patients with chronic obstructive pulmonary disease under spinal anesthesia and general anesthesia. BMC Surg. 18, 1:65. doi: 10.1186/s12893-018-0396-1

Cengiz, Y, Janes, A, Grehn, A, Israelson, LA, 2005: Randomized clinical trial of traditional dissection with electrocautery versus ultrasonic fundus-first dissection in laparoscopic cholecystectomy. Br. J. Surg. 92, 7:810-3.

Elshaer, M, Gravante, G, Thomas, K, Sorge, R, Al-Hamali, S, et al, 2015: Subtotal cholecystectomy for difficult gallbladders: Systematic review and meta-analysis. JAMA Surg. 150:15968 .

Gupta, A, Agarwal, PN, Kant, R, Malik, V, 2004: Evaluation of fundus-first laparoscopic cholecystectomy. JSLS 8, 3:255-8.

Halpin, V, 2014: Acute cholecystitis. BMJ Clin. Evid. 2014. pii: 0411.

Henneman, D, da Costa, DW, Vrouenraets, B C, van Wagensveld, BA, Lagarde, SM, 2013: Laparoscopic partial cholecystectomy for the difficult gallbladder: a systematic review. Surg. Endosc. 27:351-8.
Hugh, TB, 2002: New strategies to prevent laparoscopic bile duct injury-surgeons can learn from pilots. Surgery 132:826-35.

Hugh, TB, Kelly, MD, Mekisic, A, 1997: Rouviere's sulcus: A useful landmark in laparoscopic cholecystectomy. Br. J. Surg. 84, 9:1253-4.

Hunter, JG, 1991: Avoidance of bile duct injury during laparoscopic cholecystectomy. Am. J. Surg. 162:71-6.

Ichihara, T, Takada, M, Ajiki, T, Fukumoto, S, Urakawa, T, et al, 2004: Tape ligature of cystic duct and fundus-down approach for safety laparoscopic cholecystectomy: Outcome of 500 patients. Hepatogastroenterology 51:362-4.

Kato, K, Kasai, S, Matsuda, M, Onodera, K, Kato, J, et al, 1996: A new technique for laparoscopic cholecystectomy-retrograde laparoscopic cholecystectomy: An analysis of 81 cases. Endoscopy 28:356-9.

Koperna, T, Kisser, M, Schulz, N, 1999: Laparoscopic versus open treatment of patients with acute cholecystitis. Hepatogastroenterology 46: 753-7.

Lo, CM, Fan, ST, Liu, CL, Lai, EC, Wong, J, 1997: Early decision for conversion of laparoscopic to open cholecystectomy for treatment of acute cholecystitis. Am. J. Surg. 173:513-7.

Mahmud, S, Masaud, M, Canna, K, Nassar, AHM, 2002: Fundus-first laparoscopic cholecystectomy. Surg. Endosc. 16:581-4.

Mattioli, FP, Cagnazzo, A, Razzetta, F, Bianchi, C, Varaldo, E, et al, 1999: Laparoscopic cholecystectomy; An analysis of the reasons for a conversion to conventional surgery in an elective surgery department. Minerva Chir. 54:4716.

McIntyre, RC, Jr, Bensard, DD, Stiegman, G V, Pearlman, NW, Durham, J, 1996: Exposure for laparoscopic cholecystectomy dissection alters biliary ductal anatomy. Surg. Endosc. 10:413.

Metwaly, HA, Al-Gayyar, MM, Eletreby, S, Ebrahim, MA, El-Shishtawy, MM, 2012: Relevance of serum levels of interleukin-6 and syndecan-1 in patients with hepatocellular carcinoma. Sci. Pharm. 80, 1:179-88

Mouret, G, 1991: From the first laparoscopic cholecystectomy to the frontiers of laparoscopic surgery: The prospective futures. Dig. Surg. 8: 124-5

Neri, V, Lapolla, F, Forlano, I, Di Lascia, A, Fersini, A, et al, 2013: Cholecystectomy morbi- 
dity in the laparoscopic era. Wyno J. Med. Sci. 2, 2:S9-25

Ota, A, Kano, N, Kusanagi, H, Yamada, S, Garg, A, 2003: Techniques for difficult cases of laparoscopic cholecystectomy. J. Hepatobil. Pancreat. Surg. 10:172-5.

Pananivelu, C, Rajan, PS, Jani, K, Shetty, A

R, Sendhilkumar, K, et al, 2006: Laparoscopic cholecystectomy in cirrhotic patients: the role of subtotal cholecystectomy and its variants. J. Am. Coll. Surg. 203, 2:145-51.

Parra Blanco, JA, Bueno López, J, Madrazo Leal, C, Fariñas Alvarez, C, Torre Carrasco, F, et al, 1999: Laparoscopic cholecystectomy: analysis of risk factors for predicting conversion to open cholecystectomy. Rev. Esp. Enferm. Dig. 91:359-64.

Raj, PK, Castillo, G, Urban, L, 2001: Laparoscopic cholecystectomy: fundus-down approach. J. Laparoendosc. Adv. Surg, Tech.11, 2:95100.

Reynolds, W, Jr, 2001: The first laparoscopic cholecystectomy. JSLS 5:89-94.

Rosenberg, J, Leinskold, T, 2004: Dome down laparoscopic cholecystectomy. Scand. J. Surg. 93:48-51.

Sekimoto, M, Tomita, N, Tamura, S, Ohsato, H, Monden, M, 1998: New retraction technique to allow better visualization of the Calot's traingle during laparoscopic cholecystectomy. Surg. Endosc. 12:1439-41.

Strasberg, SM, 2002: Avoidance of the biliary injury during laparoscopic cholecystectomy. J. Hepatobil. Pancreat. Surg. 9:543-7.

Strasberg, SM, Dirk, DJ, 2012: Extreme vasculo-biliary injuries: association with fundus-down cholecystectomy in severely inflamed gallbladders. J. HPB Surg. 14, 1:1-8

Tuveri, M, Calo, PG, Medas, F, Tuveri, A, Nicolosi, A, 2008: Limits and advantages of fundus-first laparoscopic cholecystectomy: Lessons learned. J. Laparoendosc. Adv. Surg. Tech. 18: 69-75.

Wang, YC, Yang, HR, Chung, PK, Jeng, LB, Chen, RJ, 2006: Role of fundus-first cholecystectomy in the management of acute cholecystitis in elderly patients. J. Laparoendosc. Adv. Surg. Tech.16, 2:124-7.

Wolf, AS, Nijsse, BA, Sokal, SM, Chang, Y, Berger, DL, 2009: Surgical outcomes of open cholecystectomy in the laparoscopic era. Am. J. Surg. 197:781-4.

Yamakawa, T, Zhang, T, Midorikawa, Y, Ishiyama, K, Sugiyama, Y, 2007: A case of cystic duct drainage into the left intrahepatic duct and the importance of laparoscopic fundus-first cholecystectomy for the prevention of bile duct injury. J. Laparoendosc. Adv. Surg. Tech.17, 5: 662-5.

Zha, Y, Chen, XR, Luo, D, Jin, Y, 2010: The prevention of major bile duct injures in laparoscopic cholecystectomy: Diagnostic and experience with 13,000 patients in a single center. Surg. Laparosc. Endosc. Percutan. Tech. 20:378-83. 